\title{
Identifying magnetic response of split-ring resonators at microwave frequencies
}

\author{
K. AYDIN*1,2 and E. OZBAY ${ }^{1,2,3}$ \\ ${ }^{1}$ Nanotechnology Research Centre, Bilkent University, Bilkent, 06-800 Ankara, Turkey \\ ${ }^{2}$ Department of Physics, Bilkent University, Bilkent, 06-800 Ankara, Turkey \\ ${ }^{3}$ Department of Electrical and Electronics Engineering, Bilkent University, Bilkent, 06-800 Ankara, Turkey
}

\begin{abstract}
In this study we provide experimental methods to identify the magnetic resonance of split ring resonators (SRR) at the microwave frequency regime. Transmission measurements were performed on both single SRR unit cell and periodic arrays of SRRs. The magnetic response of the SRR structure was demonstrated by comparing the transmission spectra of SRRs with closed ring resonators (CRR). Effects of the changes in the effective dielectric constant of the SRR medium on the band-gaps of SRR are investigated experimentally. SRRs not only exhibit a magnetic resonance band gap but also a band gap due to the electric resonance. Finally, we present the effect of electric coupling to the magnetic resonance of bianisotropic SRRs by utilizing SRRs with different orientations, and incident electromagnetic wave polarizations.
\end{abstract}

Keywords: split ring resonator, negative permeability, left-handed materials, metamaterials.

\section{Introduction}

The realization of media that exhibited the effective negative index of refraction $n_{\text {eff }}$ made a great impact on the scientific community and received a considerable amount of interest recently. This in turn resulted in the discovery of various novel aspects of left-handed electromagnetism. Inspired by the proposal of Veselago in 1968 [1], an intuitive approach was taken to construct a composite medium, where the components simultaneously possess negative permittivity $\varepsilon<0$ and negative permeability $\mu<0$, over a certain frequency range [2]. In such a case, the wave vector of the propagating electromagnetic (EM) wave would be real and $n_{\text {eff }}$ would be negative [3]. Periodically arranged thin metallic wire structures are shown to exhibit the plasma frequency $\omega_{p}$ in the microwave regime, below which the material is opaque [4]. Since the dielectric permittivity becomes negative below $\omega_{p}$, thin wire arrays are good candidates for the negative $\varepsilon$ component of composite materials. Later, Pendry et al. proposed split-ring resonator (SRR) structures and demonstrated that such structures strongly respond to an incident magnetic field resulting in negative permeability near the magnetic resonance frequency $\omega_{m}$ [5]. The experimental verification of negative refraction is reported for such composite materials of SRRs and wires, supporting the existence of the negative refractive index $n_{\text {eff }}<0$ medium [3,6,7]. These structures are called left-handed metamaterials (LHM), attributing to the left-handed (LH) coordinate system formed by the EM wave components in the medium. In such media, the elec-

\footnotetext{
*e-mail: aydin@fen.bilkent.edu.tr
}

tric, magnetic, and wave vector components do not obey the right-hand rule [1].

Split ring resonators are responsible for the negative permeability part of the negative refraction. The discovery of SRR structures by Pendry, enabled the possibility of obtaining left-handed materials. Since SRR structures are quite undiscovered and they are the essential components of LHMs for the time being, there are extensive studies in the literature regarding the various aspects of SRR structures. Transmission characteristics of periodic [8-11] and disordered SRRs [12] are studied experimentally in detail. Several works reported the numerical simulations of the transmission properties [13-16], and the effective parameters [17,18] of SRRs. Electromagnetic resonance properties of SRRs are studied extensively in both theoretical and experimental manners[19-21]. Analytical models are reported to understand the origin of resonances and the effect of several parameters on SRRs' resonance behaviours [22-26]. The studies on SRRs and metamaterials are mainly performed at the gigahertz $(\mathrm{GHz})$ frequency regime because of their ease of fabrication, but recently there has been a tendency to increase the magnetic resonance frequencies of SRRs up to terahertz (THz) frequencies [27-30].

In this paper, we describe the methods for identifying the magnetic response of split-ring resonators at microwave frequencies. At the beginning, the split-ring resonators used in the experiments and experimental setups are introduced to the reader. In the results and discussion sections, we discuss four different ways for verifying the magnetic response of SRR structures. First, we measured the transmission through a single SRR unit cell and observed the resonant behaviour as a dip in the transmission spec- 
trum. Second, we made use of closed ring resonators to destroy the magnetic resonance. Third, we changed the effective dielectric constant of the SRR medium and therefore affected the electric response rather than the magnetic response. The band-gap due to magnetic resonance was shown to be unaffected but the band gap due to electric resonance changed. Lastly, we showed electric coupling to the magnetic resonance of SRRs. The transmission was measured for the different polarization of EM waves and the different orientation of SRRs.

\section{Spilt-ring resonator structures}

The SRR structure [3] proposed by Pendry et al. is commonly used in LHM studies. This structure consists of two concentric rings separated by a gap, both of which have splits at the opposite sides [Fig. 1(a)]. LHMs are then fabricated as periodic structures of the alternating layers of SRRs and wires. In this study we focus on the magnetic response of SRR structures since they play an important role by providing the negative permeability component to the left-handed behaviour. Figure 1(a) shows a schematic drawing of a unit cell of SRR. The dark patterns belong to metal and the lighter patterns belong to the dielectric board. Metallic patterns are printed on FR4 dielectric printed circuit boards (PCB). 30- $\mu \mathrm{m}$ thick copper is used for deposition. The dielectric board has a thickness of $1.6 \mathrm{~mm}$ and dielectric constant of $\varepsilon=3.85$. The width of the splits and the gap between the inner and outer rings are $0.2 \mathrm{~mm}$, the metal width is $0.9 \mathrm{~mm}$ and the outer radius is $3.6 \mathrm{~mm}$. The directions are provided in the figure. If the SRR structure is rotated along the $z$-axis by $90^{\circ}$, the SRR structure given in Fig. 1(b) is obtained.

SRR structures are subsequently arranged periodically to obtain negative permeability. The periodicity along the $x$ and $y$ directions is achieved by printing the 2D-arrays of SRR patterns on planar PCB boards. The periodicity in the $x$ and $y$ directions are $a_{x}=a_{y}=8.8 \mathrm{~mm}$. The periodicity in the $z$ direction is achieved by stacking a large number of such patterned planar substrates. The planar SRR boards
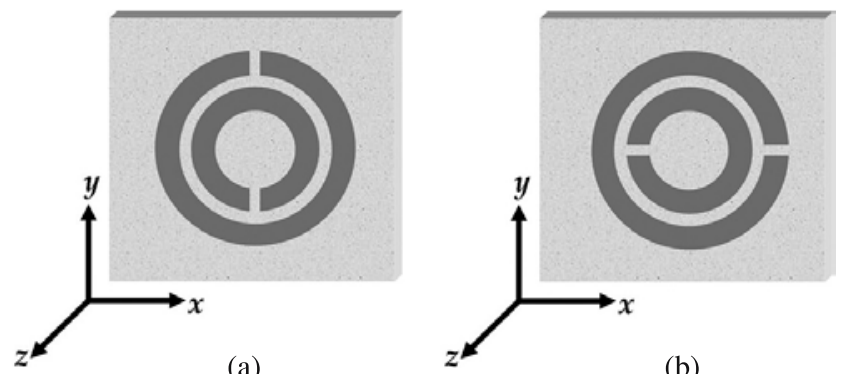

(a)

(b)

Fig. 1. Single unit cell of split-ring resonators with a split-axis along the (A) $y$ direction, (B) $x$ direction.

are stacked with a lattice constant of $a_{z}=6.5 \mathrm{~mm}$ along the $z$-axis. Notice that there is a $4.9 \mathrm{~mm}$ air region between the neighbouring SRR boards.

\section{Experimental setups}

Two different experiments were performed in this study. One was the measurement of the transmission coefficients of a single SRR unit cell, and the other was that of a periodic array of SRRs and related structures. In order to determine the magnetic resonance frequency $\omega_{m}$, we measured the transmission through a single unit cell of SRR and CRR. Two monopole antennas were used to transmit and detect the EM waves through the single SRR unit cell. The monopole antenna was constructed by removing the shield around one end of a microwave coaxial cable. The exposed centre conductor, which also acted as the transmitter and receiver, was $3.8 \mathrm{~mm}$ long. This length is on the order of $\lambda / 2$, arranged to work at a frequency range covering the $\omega_{m}$ of the SRR structures. A single SRR was placed between the monopole antennas as shown in Fig. 2(a). The distance between the monopole and SRR unit cell is $6 \mathrm{~mm}$. Monopole antennas were then connected to the HP-8510C network analyzer in order to measure the transmission coefficients. First, we measured the transmission spectra in free space (i.e., without SRR unit cell), which is used as the calibration data for the network analyzer. Then, a SRR unit cell was inserted between the monopole antennas, in which

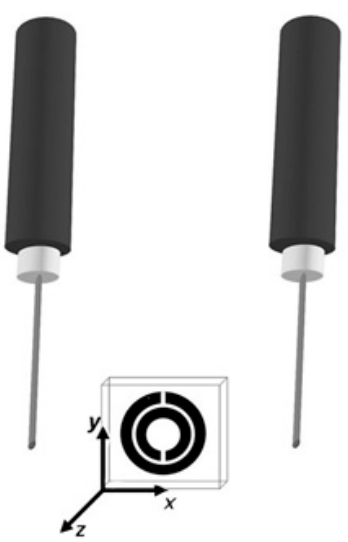

(a)
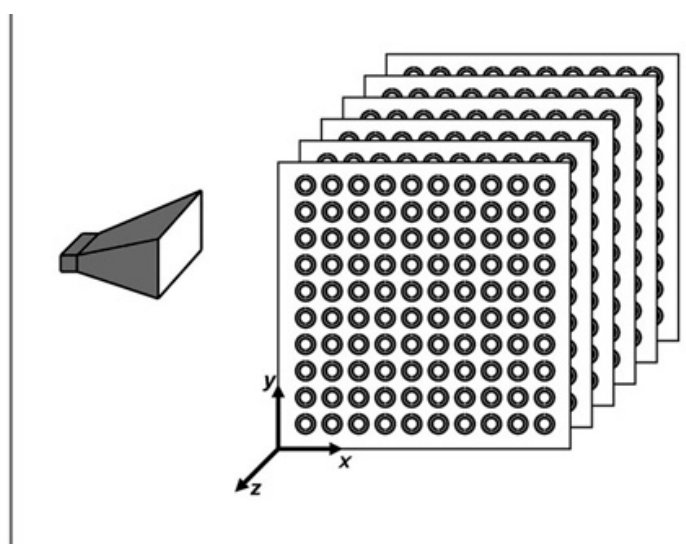

(b)

Fig. 2. Experimental setups for measuring the transmission coefficient of (a) periodic SRR array, and (b) single unit cell of SRR structure. 
we performed the transmission measurements by maintaining the distance between the transmitter and receiver monopole antennas fixed.

For measuring the transmission through the periodic SRR arrays, standard high-gain microwave horn antennas were connected to the HP $8510 \mathrm{C}$ network analyzer. One of the horn antennas transmitted the electromagnetic (EM) wave and the other one detected the transmitted EM wave. The transmission spectrum in free space was measured and set as the calibration data for the network analyzer. Then, the structure was inserted between the horn antennas, in which the transmission measurements were performed. The distance between the horn antennas was maintained at 35 $\mathrm{cm}$ to ensure that the near fields would not have considerable effects on the transmission. Both transmission measurements were performed in free space (air) rather than a waveguide environment.

\section{Results and discussions}

Before we commence to report these methods, we need to mention that the SRR structures that were used in this study were previously reported to exhibit left-handed behaviour when combined together with a proper thin wire medium [6,9]. The LHM structure composed of these SRRs also have a negative index of refraction [6,7]. However, in this study we aimed to show the magnetic response of SRRs without making use of thin wire structures, but rather focusing only on the SRR structures. The magnetic response of SRRs also could be verified by using theoretical methods such as retrieval procedures and determining the field patterns inside the SRR structure. This study, however, brings attention to the experimental methods. This section is divided into 4 subsections; in each subsection we show a different method to identify the magnetic response of SRR structures.

\subsection{Magnetic response of SRR unit cell}

The SRR structure is strongly resonant around the magnetic plasma frequency $\omega_{m}$ [5], which is induced by the currents and split, which imitates magnetic poles. This resonant behaviour is due to capacitive elements such as gaps and splits, and in turn results in very high positive and negative values of permeability close to $\omega_{m}$. If a single SRR unit cell is excited with an EM wave with the appropriate polarization, the SRRs give a strong response to the magnetic component of the incident field due to the magnetic resonance. This resonant behaviour of SRRs can be experimentally observed by measuring the transmission through SRRs. Subsequently, a dip in the transmission spectrum of the SRR structure can be attributed to the resonant nature of SRRs [20].

We measured the transmission through SRRs for two different orientations as seen in Fig. 1. Imagine a line that connects two splits and for the convention we name this imaginary line as split-axis. For the SRR (A) structure a split-axis is parallel to the $y$-axis, however, for the SRR (B) structure it is parallel to the $x$-axis. The incident EM wave propagates along the $x$ direction, with an electric field $\mathbf{E}$ and magnetic field $\mathbf{H}$ along the $y$ and $z$ directions, respectively. For such a polarization, the magnetic field is perpendicular to the SRR plane (located at $x-y$ plane) and therefore excites the magnetic resonance of SRRs for both orientations.

Figure 3(a) shows the measured transmission spectra for single SRR (A) and SRR (B) structures. Both of the SRR structures have dips at the transmission spectra at a frequency of $3.82 \mathrm{GHz}$. However, the dip values are quite different for these SRRs. The SRR (A) structure has a dip value of $-28 \mathrm{~dB}$, on the other hand, the dip value for the SRR (B) is $-7 \mathrm{~dB}$. Therefore, the orientation of the SRR structure with respect to an electric field may affect the strength of the resonance. This reduction in dip value for the SRR (B) structure can be explained by an electric coupling to the magnetic resonance, which will be explained in sec. 4.4 .

Notice that the wavelength is $7.86 \mathrm{~cm}$, whereas the diameter of the SRR structure is $7.2 \mathrm{~mm}$. Therefore, by employing the aforementioned SRR structure with sizes corresponding to the $1 / 11^{\text {th }}$ of free space wavelength, magnetic resonance can be obtained. The wavelength in the material $(4.0 \mathrm{~cm})$ is even approximately $1 / 6^{\text {th }}$ of structure size. The reason why SRR received a great amount of interest is not only because of being a source for negative permeability, but also for achieving resonance with subwavelength features. Therefore, an effective medium theory becomes applicable for SRR media, and LHMs.

\subsection{Closed ring resonator structure for verifying magnetic resonance}

The splits in the SRR structure play a key role for obtaining magnetic resonance. Removing the splits will prevent current from flowing between the inner and outer rings [21]. Since there is not any more current flowing between the concentric rings, the magnetic resonance will be destroyed [9]. We have employed a structure where the splits at the rings of the SRR are removed. The resulting structure is then two concentric rings separated by a gap. As a convention we call this structure a closed ring resonator (CRR). The measured transmission spectra of a single CRR unit cell are provided in Fig. 3(a) with dashed lines. Throughout the frequencies of interest, the incident EM wave is completely transmitted. This clearly proves that the resonance observed in sec. 4.1 is of magnetic origin.

The SRR structure does not only exhibit magnetic resonance induced by the splits at the rings. Electric resonance is also present via the dipole-like charge distribution along the incident electric field. Figure 3(b) shows the frequency response of single SRR at higher frequencies between 8-12 GHz. Evidently, we have observed sharp dip for SRR (A) and CRR structure at the same frequency, $10.6 \mathrm{GHz}$. 


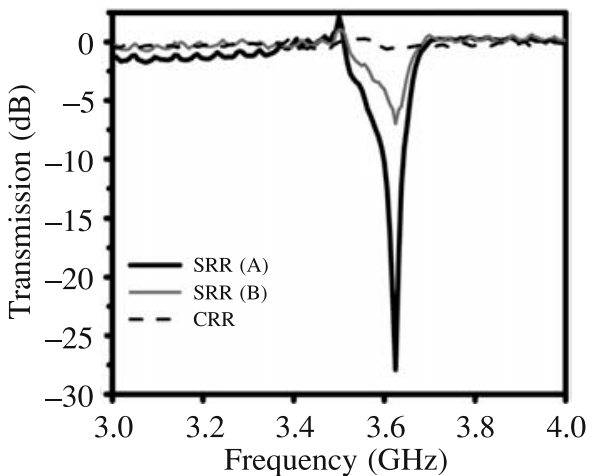

(a)

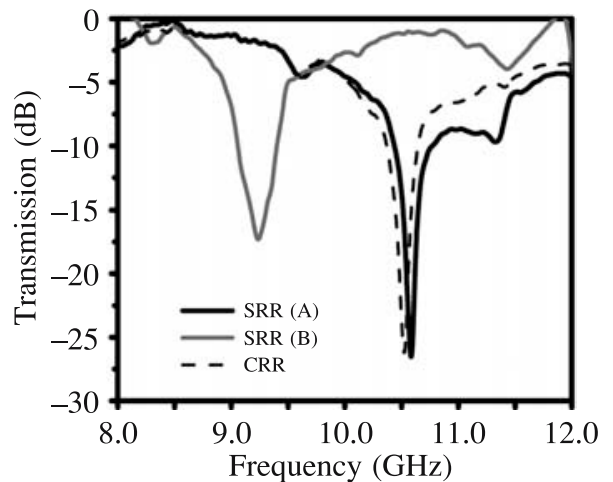

(b)

Fig. 3. Measured transmission spectra of single unit cell of SRR (A), SRR (B) and CRR structures (a) between 3-4 GHz and (b) between $8-12 \mathrm{GHz}$.

Closing the split did not destroy the electrical resonance for SRR (A) structure. Interestingly, the electrical resonance frequency for SRR (B) structure is observed at a lower frequency, 9.2 GHz. One should expect the electrical resonance to be at same frequencies for all structures, however, the behaviour is different for SRR (B) structure. We attribute this behaviour to the orientation of the splits with respect to the electric field. In SRR (A) structure, the electric field does not see the splits, however, in SRR (B) case the electric field does indeed see the splits. This results a downward shift in the electrical resonance of the SRR.

A band gap in the transmission spectrum of a periodic SRR medium may be due to the negative $\varepsilon$ or negative $\mu$, or solely due to the periodicity. The ambiguity can be lifted by using the CRR structure. This will destroy the magnetic resonance but retain the electric resonance. A frequency gap that is present in the transmission spectrum of SRR medium but not in the CRR medium will correspond to $\mu<0$. We have measured transmission through the periodic arrays of SRR and CRR structures. The number of unit cells along the $x, y$, and $z$ directions are $N_{x}=10, N_{y}=20$, and $N_{z}=25$. The incident EM wave propagates along the $x$ direction, while $\mathbf{E}$ is along the $y$ direction, and $\mathbf{H}$ is along the $z$ direction [see Fig. 2(b) for the directions]. Figure 4 shows the measured transmission spectra of periodic SRRs (solid line) and CRRs (dashed line) between 3-13 GHz. The results provided in Fig. 4 belong to the SRR (A) configuration. The first band gap in the transmission spectrum of the SRR medium between $3.55-4.05 \mathrm{GHz}$ is not present in that of the CRR medium. However, the second band gap $(8.3-12.0 \mathrm{GHz})$ is present for both the SRR and CRR arrays. Therefore, the first band gap is due to the magnetic resonance induced by the splits and thus permeability becomes negative throughout the band gap. This measurement clearly shows that the stop bands of an SRR medium cannot be automatically assumed as a result of "negative $\mu$ " behaviour. Some of the observed gaps (such as a second band gap in this measurement) in the transmission spectra could also originate from the electrical response of the SRRs or from Bragg gaps due to periodicity. There is another band gap above $13 \mathrm{GHz}$ (data not shown here), where the structure size and lattice constant becomes comparable to the wavelength and thus periodic effects becomes dominant in determining the band gap.

\subsection{Changing effective dielectric constant of SRR medium}

In the previous section we investigated the transmission characteristics of SRR by removing the splits of the rings. The origins behind the formation of the first and second band gap of SRRs were shown to be different. The first band gap is due to magnetic resonance, whereas the second band gap is the result of electric resonance. SRRs, in addition to their resonant magnetic response at $\omega_{m}$, exhibit a second resonant response at the higher frequency $\omega_{o}$ which has an electrical character [18]. The behaviour is similar to that of a periodic cut-wire medium that exhibits a stop band with a well-defined lower edge due to its discontinuous wire geometry [8]. In this section we investigate this electric resonance by changing the effective dielectric constant of the SRR medium.

We first increased the lattice spacing between the SRR planes, i.e., along the $z$-direction. We chose the structure in sec 4.2 as the reference structure which has a lattice constant of $a_{z}=6.5 \mathrm{~mm}$. We increased the lattice spacing to

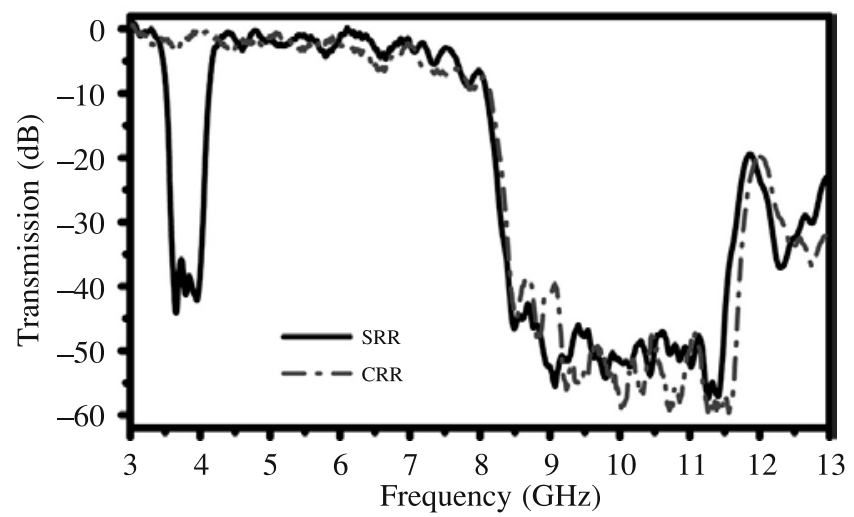

Fig. 4. Measured transmission spectra of periodic arrays of SRR (solid line) and CRR (dashed-dotted line) structures. 
$a_{z}=11.0 \mathrm{~mm}$. The effective dielectric constant of this new SRR array is decreased since the unit cell is filled with more air compared to the reference SRR array. The measurements in this section are performed by using arrays composed of SRR(A) configuration. The measured transmission spectra for SRRs with different lattice spacings are plotted in Fig. 5(a). The first band gap is present for both cases, although the lattice constant is increased approximately on the order of 2 . On the other hand, the second band gap is changed with an increase in the lattice constant. The second band gap for SRRs with $a_{z}=6.5 \mathrm{~mm}$ (solid) is between 8.3-12.0 GHz, whereas for SRRs with $a_{z}=11.0$ $\mathrm{mm}$ (dashed) the second band gap between $8.6-11.3 \mathrm{GHz}$ becomes narrower.

As pointed out in sec. 2, the SRRs are deposited on a dielectric board of thickness $w=1.6 \mathrm{~mm}$. We have increased the thickness of the dielectric board by a factor of 2 , and obtained SRR planes with $w=3.2 \mathrm{~mm}$ thickness. Lattice spacing is kept at $a_{z}=6.5 \mathrm{~mm}$, therefore almost half of the unit cell is now filled with a dielectric board. The comparison in the transmission spectra of SRRs with different thicknesses of dielectric board are provided in Fig. 5(b). The first band gap remains unaffected, but there is a downward shift in the second band gap for SRRs with $w=$ $3.2 \mathrm{~mm}$ thick dielectric (dashed). The new frequency range of the band gap is 7.8-11.1 GHz.

As discussed in sec. 4.1, single SRR is resonant at a very narrow frequency range. Arranging SRR structures periodically in all directions increases the coupling between the SRRs, therefore, a wider band gap in the transmission spectrum is observed compared to that of single SRR. It is worth mentioning that changing the effective dielectric constant of the medium does not affect the magnetic resonance band gap. The coupling of SRRs mainly occurs in the SRR plane. The effect of inter-plane coupling between the neighbouring SRR planes is not as dominant as intra-plane coupling.

\subsection{Observation of electric coupling to magnetic resonance of SRRs}

The first band gap in the transmission spectra of the SRR structure as discussed in sec. 4.2 was mentioned to be a fact of magnetic resonance excitation by magnetic field. However, it has been recently argued that for the specific orientation of SRR and polarization of an incident EM wave, it is possible to obtain a resonance due to electric field excitation $[10,21]$.

In this section we show the cross-polarization effect of the current SRR design. We have performed transmission measurements on periodic arrays of SRR structures. Four different measurements are performed for combinations of different orientations [(A) and (B) in Fig. 1] and different polarizations (parallel and perpendicular polarization). Notice that magnetic permeability is only achievable via parallel polarization, where the wave vector is parallel to the

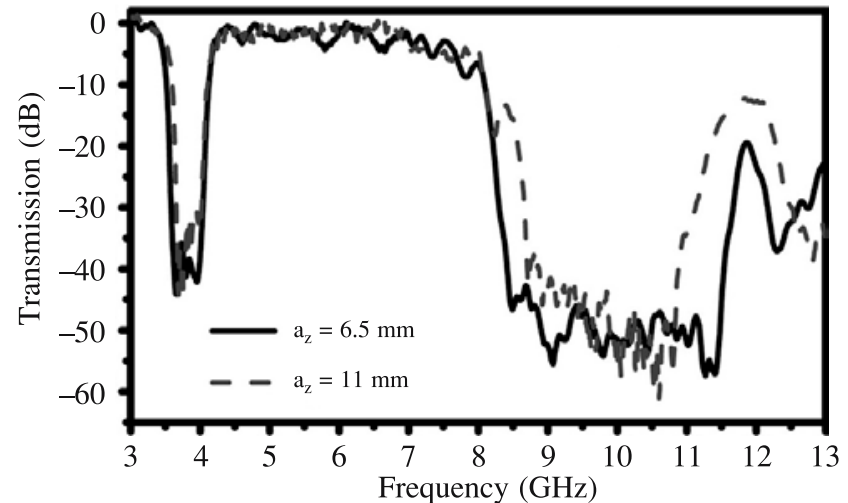

(a)

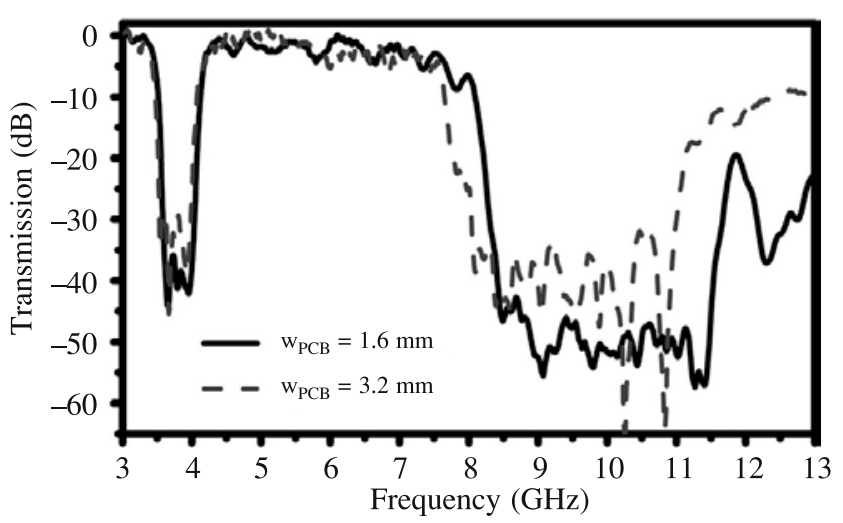

(b)

Fig. 5. Measured transmission spectra of (a) periodic arrays of SRR structures with lattice spacing along $\mathrm{z}$ directions $a_{z}=6.5 \mathrm{~mm}$ (solid) and $a_{z}=11.0 \mathrm{~mm}$ (dashed), and (b) periodic arrays of SRR structures with PCB board thickness $w_{P C B}=1.6 \mathrm{~mm}$ (solid) and $w_{P C B}=3.2 \mathrm{~mm}$ (dashed).

SRR plane and the $\mathbf{H}$-field is along the $z$-axis. A magnetic field perpendicular to the SRR induces current loops at the resonance. In sec. 4.1, we investigated the resonance due to the single unit cell of SRR (A) and (B) structures for parallel polarization. Figure 6 gives the response of a periodic array of such structures to the incident plane wave. Both structures have a band gap at the transmission spectra. For the SRR (B) structure, a wider band gap is observed between 3.5-4.3 GHz. The band gap is narrower for the SRR (A) array with a range of $3.55-4.05 \mathrm{GHz}$. Since the wave vector is parallel to the SRR plane, the type of polarization is called parallel polarization for the convention.

For the perpendicular polarization case, the wave vector is orthogonal to the SRR plane (along $z$-axis), the E-field is along the $y$-axis and the $\mathbf{H}$-field is along the $x$-axis. In this case, the magnetic field cannot excite a magnetic resonance since it is now parallel to the SRR plane. However, an electric field can induce current loops for SRR (B) orientation. We measured the transmission through a SRR periodic array with the number of unit cells along the $x, y$, and $z$ directions at $N_{x}=20, N_{y}=20$, and $N_{z}=10$. A band gap between $3.86-4.20 \mathrm{GHz}$ was observed in the transmission spectra of the SRR (B) structure for perpendicular polarization, whereas the incident waves were completely transmitted 


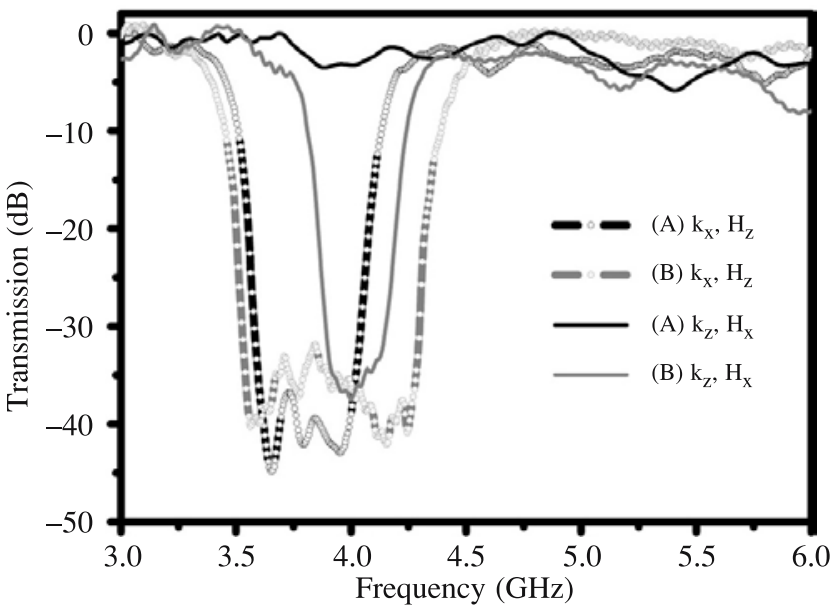

Fig. 6. Measured transmission spectra of SRR (A) structure with incident electromagnetic wave propagating along the $x$ direction with a magnetic field along the $z$-direction (bold black line with circles), SRR (B) structure with $k_{x}$ and $H_{z}$ (bold gray line with circles), SRR (A) structure with $k_{z}$ and $H_{x}$ (black solid line), and SRR (B) structure with $k_{z}$ and $H_{x}$ (gray solid line).

for SRR (A) structure. In this case the SRR's mirror symmetry is no longer present with respect to the electric field direction; because of the orientation of the splits [10]. This structure has cross-polarization effects rendering the magnetic resonance for a certain orientation [21]. This is a fact of bianisotropic behaviour of Pendry's original SRR design. There were several attempts in literature to obtain nonbianisotropic SRR designs [22,23,31].

This electric coupling to the magnetic resonance effect is a useful tool for obtaining magnetic responses of bianisotropic SRRs at higher frequencies such as $\mathrm{THz}$ frequencies [27-29]. For parallel polarization one needs to stack lots of SRR samples and therefore much more time is required to fabricate those structures. Additionally, the misalignment will become an important issue at those frequencies [12]. However, 5 to 10 samples are sufficient to observe the aforementioned cross-polarization effect by making use of the perpendicular polarization.

\section{Conclusions}

To conclude, we performed transmission measurements on split-ring resonator structures. The transmission through a single unit cell, and the periodic arrays of SRRs were measured and interpreted. A single unit cell of SRR was shown to be strongly resonant at a certain magnetic resonance frequency with a dip value of $-28 \mathrm{~dB}$ at the transmission spectra. The closed ring resonator structure is proposed to verify the magnetic response of the SRRs. The band gap disappeared for the transmission spectrum of CRR, indicating the magnetic response, thus the negative permeability of SRRs. The magnetic resonance band gap of SRRs were shown to be unaffected by the changes in the effective dielectric constant of the SRR medium. The electric resonance band gap, however, is affected considerably with the change in the dielectric constant of the medium. Finally, we observed the cross-polarization effect of SRR structures. For certain orientation of the bianisotropic SRRs and the perpendicular polarization to the SRR plane, the electric field couples to the magnetic resonance and renders the negative permeability together with the negative permittivity.

\section{Acknowledgements}

This work is supported by the European Union under the projects EU-DALHM, EU-NOE-METAMORPHOSE, EUNOE-PHOREMOST, and TUBITAK under Project No. 104E090. One of the authors (Ekmel Ozbay) also acknowledges partial support from the Turkish Academy of Sciences.

\section{References}

1. V.G. Veselago, "The electrodynamics of substances with simultaneously negative values of permittivity and permeability", Sov. Phys. Usp. 10, 509-514 (1968).

2. D.R. Smith, W.J. Padilla, D.C. Vier, S.C. Nemat-Nasser, and S. Schultz, "Composite medium with simultaneously negative permeability and permittivity”, Phys. Rev. Lett. 84, 4184-4187 (2000).

3. R.A. Shelby, D.R. Smith, and S. Schultz, "Experimental verification of a negative index of refraction", Science $\mathbf{2 9 2}$, 77-79 (2001).

4. J.B. Pendry, A.J. Holden, W.J. Stewart, and I. Youngs, "Extremely low frequency plasmons in metallic mesostructures", Phys. Rev. Lett. 76, 4773-4776 (1996).

5. J.B. Pendry, A.J. Holden, D.J. Robbins, and W.J. Stewart, "Magnetism from conductors and enhanced nonlinear phenomena", IEEE Trans. Microwave Theory Tech. 47, 2075-2084 (1999).

6. K. Aydin, K. Guven, C.M. Soukoulis, and E. Ozbay, "Observation of negative refraction and negative phase velocity in left-handed metamaterials", Appl. Phys. Lett. 86, 124102 (2005).

7. K. Aydin and E. Ozbay, "Negative refraction through an impedance-matched left-handed metamaterial slab", J. Opt. Soc. Am. B23, 415-418 (2006).

8. E. Ozbay, K. Aydin, E. Cubukcu, and M. Bayindir, "Transmission and reflection properties of composite double negative metamaterials in free space", IEEE Trans. Antennas Propag. 51, 2592-2595 (2003).

9. K. Aydin, K. Guven, M. Kafesaki, L. Zhang, C.M. Soukoulis, and E. Ozbay, "Experimental observation of true left-handed transmission peak in metamaterials", Opt. Lett. 29, 2623-2625 (2004).

10. N. Katsarakis, T. Koschny, M. Kafesaki, E.N. Economou, and C.M. Soukoulis, "Electric coupling to the magnetic resonance of split ring resonators", Appl. Phys. Lett. 84, 2943-2945 (2004).

11. Y. Qi, B. Hou, and W. Wen, "Band gaps from ring resonators and structural periodicity", J. Phys. D: Appl. Phys. 38, 590-595 (2005).

12. K. Aydin, K. Guven, N. Katsarakis, C.M. Soukoulis, and E. Ozbay, "Effect of disorder on magnetic resonance band gap 
of split-ring resonator structures", Opt. Exp. 12, 5896-5901 (2004).

13. P. Markos, and C.M. Soukoulis, "Numerical studies of left-handed materials and arrays of split ring resonators", Phys. Rev. E65, 036622 (2002).

14. R.W. Ziolkowski, "Design, fabrication, and testing of double negative metamaterials", IEEE Trans. Antennas Propag. 51, 1516-1529 (2003).

15. M. Kafesaki, Th. Koschny, R.S. Penciu, T.F. Gundogdu, E.N. Economou, and C.M. Soukoulis, "Left-handed metamaterials: detailed numerical studies of the transmission properties", J. Opt. A: Pure Appl. Opt. 7, S12-S22 (2005).

16. E.A. Semouchkina, G.B. Semouchkin, M. Lanagan, and C.A. Randall, "FDTD study of resonance processes in metamaterials", IEEE Trans. Microwave Theory Tech. 53, 1477-1487 (2005).

17. T. Koschny, P. Markos, D.R. Smith, and C.M. Soukoulis, "Resonant and antiresonant frequency dependence of the effective parameters of metamaterials", Phys. Rev. E68, 065602(R) (2003).

18. T. Koschny, M. Kafesaki, E.N. Economou, and C.M. Soukoulis, "Effective medium theory of left-handed materials", Phys. Rev. Lett. 93, 107402 (2004).

19. P. Gay-Balmaz, and O.J.F. Martin, "Electromagnetic resonances in individual and coupled split-ring resonators", $J$. Appl. Phys. 92, 2929-2936 (2002).

20. K. Aydin, I. Bulu, K. Guven, M. Kafesaki, C.M. Soukoulis, and E. Ozbay, "Investigation of magnetic resonances for different split-ring resonator parameters and designs", New J. Phys. 7, 168 (2005).

21. J. García-García, F. Martín, J.D. Baena, R. Marques, and L. Jelinek, "On the resonances and polarizabilities of split ring resonators", J. Appl. Phys. 98, 033103 (2005).

22. R. Marques, F. Mesa, J. Martel, and F. Medina, "Comparative analysis of edge- and broadside-coupled split ring reso- nators for metamaterial design - theory and experiments", IEEE Trans. Antennas Propag. 51, 2572-2581 (2003).

23. R. Marques, F. Medina, and R. Rafii-El-Idrissi, "Role of bianisotropy in negative permeability and left-handed metamaterials", Phys. Rev. B65, 144440 (2002).

24. B. Sauviac, C.R. Simovski, and S.A. Tretyakov, "Double split-ring resonators: Analytical modelling and numerical simulations", Electromagnetics 24, 317-338 (2004).

25. M. Shamonin, E. Shamonina, V. Kalinin, and L. Solymar, "Properties of a metamaterial element: Analytical solutions and numerical simulations for a singly split double ring", $J$. Appl. Phys. 95, 3778-3784 (2004).

26. J.D. Baena, J. Bonache, F. Martín, R.M. Sillero, F. Falcone, T. Lopetegi, M.A.G. Laso, J. García-García, I. Gil, M.F. Portillo, and M. Sorolla, "Equivalent-circuit models for split-ring resonators and complementary split-ring resonators coupled to planar transmission lines", IEEE Trans. Microwave Theory Tech. 53, 1451-1461 (2005).

27. T.J. Yen, W.J. Padilla, N. Fang, D.C. Vier, D.R. Smith, J.B. Pendry, D.N. Basov, and X. Zhang, "Terahertz magnetic response from artificial materials", Science 303, 1494-1496 (2004).

28. S. Linden, C. Enkrich, M. Wegener, J. Zhou, T. Koschny, and C.M. Soukoulis, "Magnetic response of metamaterials at 100 terahertz", Science 306, 1351-1353 (2004).

29. N. Katsarakis, G. Konstantinidis, A. Kostopoulos, R.S. Penciu, T.F. Gundogdu, M. Kafesaki, E.N. Economou, Th. Koschny, and C.M. Soukoulis, "Magnetic response of splitring resonators in the far-infrared frequency regime", Opt. Lett. 30, 1348-1350 (2005).

30. J. Zhou, Th. Koschny, M. Kafesaki, E.N. Economou, J.B. Pendry, and C.M. Soukoulis, "Saturation of the magnetic response of split-ring resonators at optical frequencies", Phys. Rev. Lett. 95, 223902 (2005).

31. I. Bulu, H. Caglayan, and E. Ozbay, "Experimental demonstration of labyrinth-based left-handed metamaterials", Opt. Exp. 13, 10238-18247 (2005). 